\title{
Geographische und onomatologische Bemerkangen zu der Liste der Väter des Konzils von 381.
}

Durch Chabots Publikation der Chronik des Patriarchen Michael von Antiochien (1166-1199) ist uns ein syrisches Verzeichnis ${ }^{1}$ ) der Väter des sog. II. ökumenischen Konzils bekannt geworden, dessen Vergleich mit den beiden lateinischen Rezensionen (Prisca und Dionysiana, wie sie gewöhnlich genannt werden) nicht ohne Wert ist.

Ich stelle hier einiges auf die Bischofs- wie die Städtenamen Bezügliche zusammen. ${ }^{2}$ )

5. Gelasios von Kēsarēa. Die Ausgaben der Konzilsakten haben Thalassius. Indessen das ist ein alter Druckfehler. Die lateinischen Handschriften haben ausnahmslos Gelasius oder Korruptelen dieses Namens, wie denn dieser Kirchenfürst unter seinem richtigen Namen auch sonst erwähnt wird. Die wahre Form bestätigt der Syrer.

Interessanter sind die Nummern 8-10.
\begin{tabular}{c|c|c} 
Prisca. & Diony siana. & \multicolumn{2}{|c}{ Michael. } \\
$\begin{array}{c}\text { 8. Priscianus Nicopo- } \\
\text { litanus }\end{array}$ & Priscianus Sevastensis & $\begin{array}{l}\text { Priskianos von Niko- } \\
\text { polis }\end{array}$ \\
9. Saturninus Sebaste- & Saturninus Squitopoli- & Sațūninos von Sebastīa
\end{tabular} nus tanus
10. Rufus Scytopolita-
nus

Le Quien, den Konzilienausgaben folgend, hatte Prisojanus als Bischof von Sebaste (0. Ch. III 651), Saturninus als Bischof von Skythopolis (a. a. 0. 687) und Rufus als Bischof von Nikopolis (a. a. 0. 594) eingetragen. Die Übereinstimmung von Prisca und Michael erweisen, daB die Bischofssitze zu vertauschen sind.

23. Pelagios, der bekannte Bischof von Laodikeia in Syrien, heiBt

1) Die syrischen Namen vorlaufig nur 1-184 S. 168-161 des syr. Textes, die vollständige Ubersetzung mit den Varianten des Ms Borgia S. 313-320 d. U.

2) Die Nummern sind die Chabots. 
in den Konzilsakten und in der Dionysiana Pegasius; indessen zahlzeiche Handschriften der Prisea und ebenso der Syrer bieten die wahre Namensform Pelagios.

47. Antiochus von Samosata heißt in der Prisca Antiochus Samasteos. Das ist wohl kein Schreibfehler, sondern entspricht genau dem syrischen Šamišt. Es wird wohl neben der edlern Form $\Sigma \alpha \mu \sigma \sigma \alpha \tau \alpha$ eine Vulgärform $\Sigma \alpha^{\prime} \mu \alpha \sigma \tau \iota \varsigma$ im Gebrauch gewesen sein.

67. In der Provinz Klein-Armenien bietet der Syrer neben den zwei bereits aus dem lateinischen Texte bekannten noch einen neuen: 'jnws d. Zbnws.

Es ist wohl zu lesen:

'Aianos von Zabanōs.

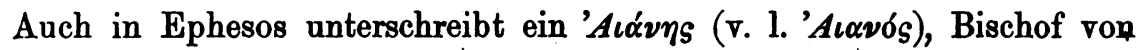
Sykamozon in Palästina. Aber rätselhaft bleibt der Stadtname. Chabòt denkt vermutungsweise an Zela; indessen dieses gehört zur Kirchenprovinz von Amasia, und nicht zu der von Melitene. Zabanōs ist wohl

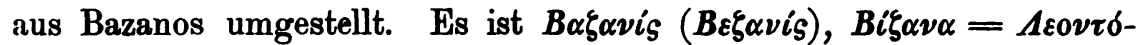

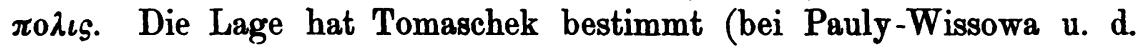
Worte Bizana). Es entspricht dem heutigen Vdžan in Derdžan (Derxene), vgl. auch Abhandl. der k. bayr. Akad. d. Wiss. I. Kl. XXI. Bd, III. Abt. S. 577.

80. Der Bischof von Trimithus heißt in den Ausgaben Theopompus, und das ist die Lesung der Dionysiana. Dagegen der Syrer hat Teoprōpos von Trimintunuțos, womit die guten Handschriften der Prisca übereinstimmen, welche Theopropus Trimitountos lesen. Der seltene,

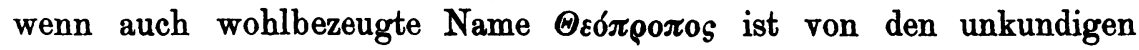

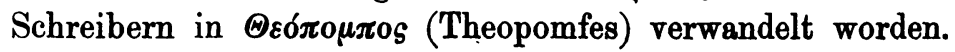

Einige Schwierigkeiten bietet Pamphylien. Hier erscheint als

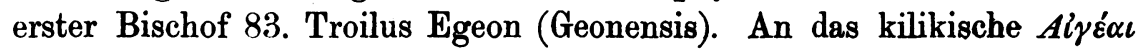
ist natürlich nicht zu denken. Auch Michael bietet Trōilōs Agion; aber der Codex des Museums Borgia hat Agānōs. Chabot denkt gewiB mit Recht an Lagina, ein Suffragenbistum des Metropoliten von

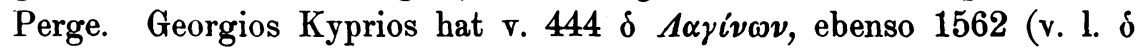
$\left.\Lambda \alpha \gamma \eta^{\prime} \omega \omega \nu\right)$. Auch Konstantinos Porphyrogennetos bietet in seiner Ek-

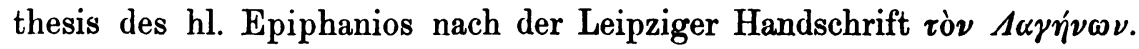
Im Quinisextum 693 unterzeichnet:

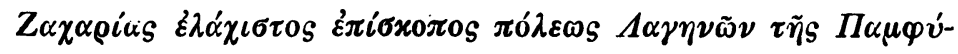

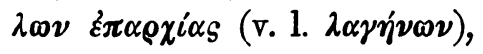

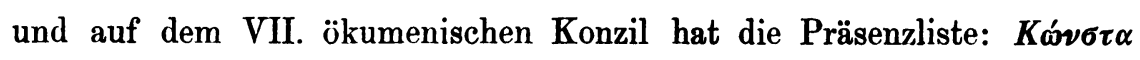
$\dot{\varepsilon} \pi$. $\Lambda \alpha \gamma \nu \tilde{\omega} \nu$ (v. l. $\Lambda \alpha \gamma i \delta \omega \nu=\Lambda \alpha \gamma i \nu \omega \nu)$. 
Er unterschreibt:

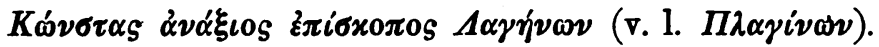

Es erscheint mir demnach unzweifelhaft, dả Agānōs für Laganos verschrieben ist. Vielleicht stand schon im Codex archetypus fehlerhaft $A \Gamma H N W N$ für $A A \Gamma H N W N$.

89 und 90 sind die Bischöfe:

Prisca.

89. Mydus Panentu

90. Heraclides Thicusitanus
Dionysiana.

Midus Panemunensis

Heraclides Tichensis
Syrer.

Mídos von Panemos

Eraḳlīīis von Tịkūs

Ramsay (the historical geography of Asia Minor S. 409) schlägt vor zu lesen: Mydus Petnelissensis, Heraclides Panemutichensis. Indéssen das widerstreitet zu sehr der Überlieferung. Die Stadt heiBt $\Pi \alpha \nu \varepsilon_{\mu o v} \tau \varepsilon \tau_{\chi 0}$, Hierocl. 681,3. Augenscheinlich entspricht 89 dem

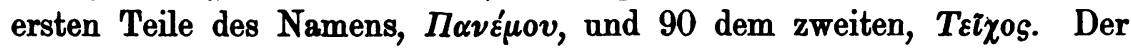
Bischof von. Panemuteichos kommt mehrfach in den Konzilsakten vor. Auch steht bisweilen $\delta \Pi \alpha \nu \varepsilon ́ \mu o v$ allein; so hat z. B. die syro-monophysitische Redaktion der Väter von Nicäa: Fausțos von Pānemōn (Patr. Nicaen. nom. S. 113). Indessen die Teilung des einen Stadtnamens in zwei Bistümer ist eine Unmöglichkeit. Offenbar liegt ein alter Fehler zu Grunde. Es handelt sich etwa um zwei Bischöfe, welche um den Sitz im Hader lagen, wie Agapios und Bagadios um Bostra. Oder der eine ist, wie so oft, der Vertreter des kranken oder altersschwachen Ordinarius. Jedenfalls gehören aber die zwei auseinander gerissenen Bestandteile des Namens zusammen.

Ein alter gemeinsamer Fehler ist auch 91 Theodolus Syalitanus der Prisca, Theodolus Sialon der Dionysiana; der Syrer hat: Teodūlos

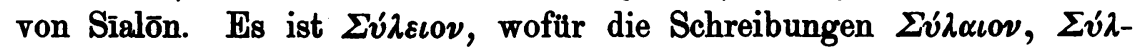
$\lambda \varepsilon \iota 0 \nu, \Sigma v i d \lambda \iota 0 \nu$ und $\Sigma i \lambda_{0 v o v}$ vorkommen.

Merkwürdig ist 111 die lateinische Schreibung Salagassitanus, Salagassensis, welche auch der Codex des Museums Borgia durch Sālāgasos bestätigt. Möglicherweise liegt ein alter Schreibfehler zu Grunde; aber die Métathese kann auch einer Vulgärform angehören, vgl. 'Avá$\beta \alpha \rho \zeta o s$ (Anavarzus) neben 'Aváł $\alpha \rho \beta o s$.

Ähnlich steht es mit dem folgenden Bischofe:

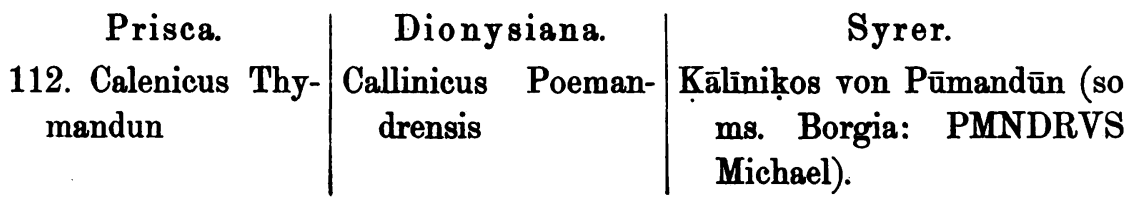




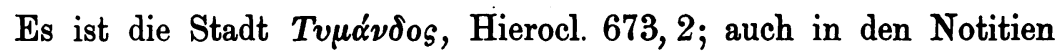

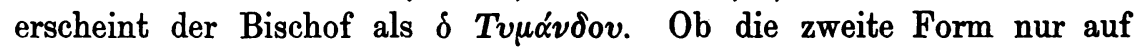
einem Schreibfehler beruht? Möglich wäre, daß neben Tv́ $\mu \alpha \nu \delta o s$ auch eine zweite Form $\Pi v \dot{v} \mu \nu \delta$ os oder $\Pi v \dot{\mu} \mu \nu \delta \rho o s$ (auch der Monacensis des

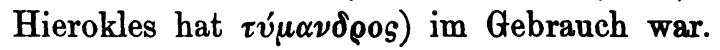

127 lautet in den Ausgaben: Romanus Bibonensis. Indessen das ist ein alter Fehler der Dionysiana. Handschriftlich heiBt der Bischof:

\begin{tabular}{c|c|cc}
\multicolumn{2}{c|}{ Prisca. } & Dionysiana. & \multicolumn{2}{|c}{ Syrer. } \\
Romanus & Pasilidun & RomanusBubonensis & $\begin{array}{c}\text { Romanos } \\
\text { von Fìsilis } \\
\text { Borgia: Fāsălōs). }\end{array}$
\end{tabular}

Es ist der Bischof von Phaselis.

Wenn so in der Hauptsache der Syrer beweist, daB die Prisca eine bessere Überlieferung als die Dionysiana zeigt, so gibt es doch auch, wenn auch weniger zahlreiche Fälle, wo die Dionysiana vereint mit dem Syrer das Richtige gegenüber der Prisca bietet.

So heiBt 15 der Bischof von Ptolemais-Akko Nëstabos nach dem Syrer und der Dionysiana, während die Prisca unrichtig Nestabius bietet. Ebenso wird für den 26. Bischof von Seleukeia die Namensform Byzus der Dionysiana gegenüber Byzas der Prisca durch des Syrers Bīzōs geschützt. Ähnlich steht es mit dem lykaonischen Bischof:

$$
\begin{array}{c|c|c}
\multicolumn{1}{c|}{\text { Prisca. }} & \text { Dionysiana. } & \text { Syrer. } \\
\text { Ininius Corinun } & \text { Inzus Coriensis } & \text { Inzos von Kọōinin. }
\end{array}
$$

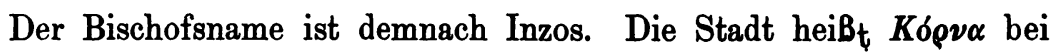
Ptolemaeus 5, 6, 16 und bei Hierocl. 676, 1. Ebenso unterschreibt in

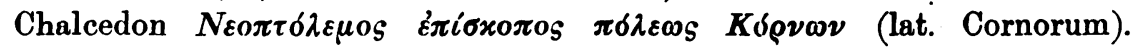

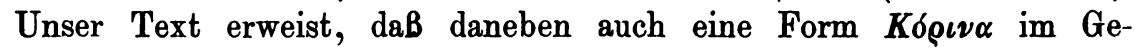
brauche war.

101. Der Bischof von Kanna ( $\delta K \alpha \nu 0 \tilde{v})$ heifst in der Prisca Eustathius; Dionysiana und Syrer verbürgen vielmehr als Namen Eustratios.

Ebenfalls nach Lykaonien gehört der Bischof:

\begin{tabular}{c|c|c} 
Prisca. & Dionysiana. & \multicolumn{2}{|c}{ Syrer. } \\
$\begin{array}{l}\text { 103. Eugenius Posa- } \\
\text { dun }\end{array}$ & $\begin{array}{l}\text { Eugenius Pasolonen- } \\
\text { sis }\end{array}$ & $\begin{array}{c}\text { Eugenios } \\
\text { von Pōsalā (Ms. } \\
\text { Borgia: Prōsālōn). }\end{array}$
\end{tabular}

Der Name der Stadt ist $\Pi \delta \sigma \alpha \lambda \alpha$; er erscheint in den ältesten

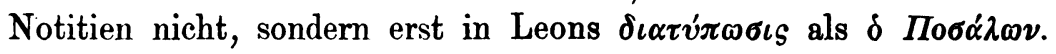


130 I. Abteil. H. Gelzer: Geographische und onomatologische Bemerkungen

Den SchluB machen wir mit einem pisidischen Bischof:

\begin{tabular}{l|c|c} 
Prisca. & Dionysiana. & \multicolumn{2}{|c}{ Syrer. } \\
$\begin{array}{l}\text { 108. Attalus Prosta- } \\
\text { dun }\end{array}$ & Attalus Prostanensis & $\begin{array}{l}\text { Attalos von Prōstānōn (so } \\
\text { Ms. Borgia). }\end{array}$
\end{tabular}

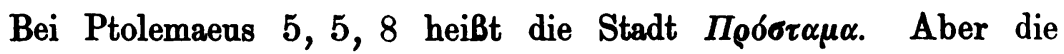

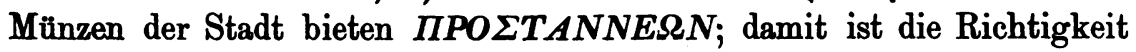
der Lesung des Syrers und der Dionysiana erwiesen. In den Notitiae fehlt das Bistum, weil es, wie Ramsay (Asia Minor S. 407) vermutet, mit einem benachbarten Bischofssitze vereinigt ward.

Diese wenigen Bemerkungen sollen nur zeigen, welchen Wert die Konzilsakten für die alte Geographie besitzen.

H. Gelzer. 